\title{
Influence of the Ringwoodite-Perovskite transition on mantle convection in spherical geometry as a function of Clapeyron slope and Rayleigh number
}

\author{
M. Wolstencroft ${ }^{1, *}$ and J. H. Davies ${ }^{1}$ \\ ${ }^{1}$ School of Earth and Ocean Sciences, Cardiff University, Main Building, Park Place, Cardiff, Wales, CF10 3AT, UK \\ *now at: University of Ottawa, Ottawa, Ontario, Canada
}

Received: 21 July 2011 - Published in Solid Earth Discuss.: 4 August 2011

Revised: 13 November 2011 - Accepted: 21 November 2011 - Published: 19 December 2011

\begin{abstract}
We investigate the influence on mantle convection of the negative Clapeyron slope ringwoodite to perovskite and ferro-periclase mantle phase transition, which is correlated with the seismic discontinuity at $660 \mathrm{~km}$ depth. In particular, we focus on understanding the influence of the magnitude of the Clapeyron slope (as measured by the Phase Buoyancy parameter, $P$ ) and the vigour of convection (as measured by the Rayleigh number, $R a$ ) on mantle convection. We have undertaken 76 simulations of isoviscous mantle convection in spherical geometry, varying $R a$ and $P$. Three domains of behaviour were found: layered convection for high $R a$ and more negative $P$, whole mantle convection for low $R a$ and less negative $P$, and transitional behaviour in an intervening domain. The boundary between the layered and transitional domain was fit by a curve $P=\alpha R a^{\beta}$ where $\alpha=-1.05$, and $\beta=-0.1$, and the fit for the boundary between the transitional and whole mantle convection domain was $\alpha=-4.8$, and $\beta=-0.25$. These two curves converge at $R a \approx 2.5 \times 10^{4}$ (well below Earth mantle vigour) and $P \approx-0.38$. Extrapolating to high $R a$, which is likely earlier in Earth history, this work suggests a large transitional domain. It is therefore likely that convection in the Archean would have been influenced by this phase change, with Earth being at least in the transitional domain, if not the layered domain.
\end{abstract}

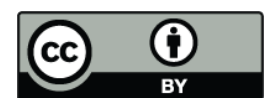

Correspondence to: J. H. Davies (daviesjh2@cf.ac.uk)

\section{Introduction}

Mantle convection has had a dominant control on Earth's surface evolution. It has been known for many years that mineral phase changes with negative Clapeyron slope $(\gamma)$, where $\gamma=d P / d T$, are capable of layering mantle convection (Olson and Yuen, 1982; Christensen, 1995). Layering occurs when the lateral temperature variations of convection produce laterally varying vertical deflections of the boundary away from its equilibrium position. The nature and magnitude of the deflections depend on the value of $\gamma$. If there is an appropriate restoring density contrast between the phases, the deflection is accompanied by buoyancy forces that act against the convective thermal buoyancy. If the Clapeyron slope and density difference are sufficient, the buoyancy forces resulting from the phase boundary deflections can overcome the local convective thermal buoyancy, resulting in layered convection (Fig. 1).

The mineral phase change in the olivine system from ringwoodite to ferro-periclase and $\mathrm{Mg}$-perovskite is known to have a negative Clapeyron slope (i.e. is an endothermic reaction) and a restoring density increase. This reaction is widely believed to correspond to the seismic discontinuity at $660 \mathrm{~km}$ depth, separating the upper and lower mantle (Bernal, 1936; Ringwood, 1969; Shim et al., 2001). Mineralogical work produces estimates of the Clapeyron slope between $-0.5 \mathrm{MPa} \mathrm{K}^{-1}$ and $-3.5 \mathrm{MPa} \mathrm{K}^{-1}$, with recent values for a dry mantle heading towards the less negative value (Katsura et al., 2003; Fei et al., 2004; Litasov et al., 2005; Hirose, 2002; Ito and Takahashi, 1989; Irifune et al., 1998). Recent measurements for a "wet" mantle have the more negative values (Ohtani and Litasov, 2006). Seismological estimates in-situ of the slope of phase change at $660 \mathrm{~km}$ depth

Published by Copernicus Publications on behalf of the European Geosciences Union. 

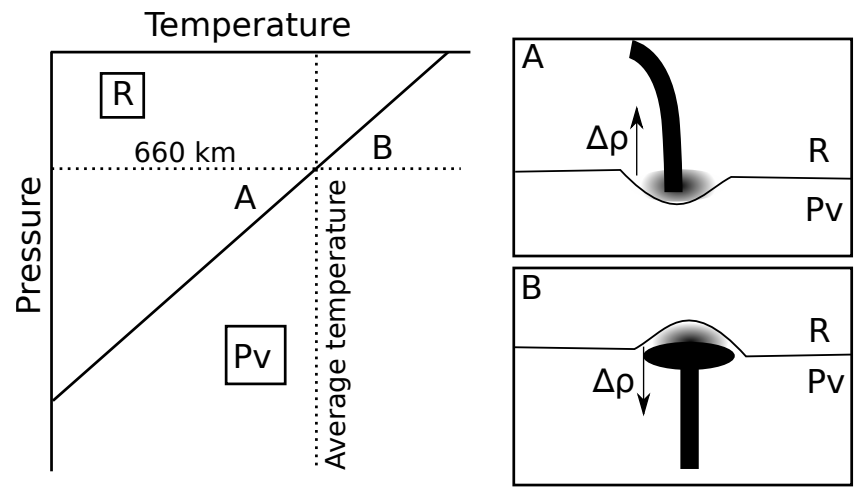

Fig. 1. Illustration of the layering mechanism modelled. The left hand side figure shows a schematic version of the phase diagram of the olivine component - Ringwoodite is stable in the upper left region (labelled with $\mathrm{R}$ ) while Perovskite and Ferropericlase is stable in the region (labelled with $\mathrm{Pv}$ ) below the phase boundary line. The right hand side: Part (A) illustrates a cold downwelling impinging on the phase change. The fact that the material below the nominal depth of the phase change can still be the lighter Ringwoodite phase is shown by the letter A on the phase diagram to the left. As a result, there is a downward deflection of the boundary, which leads to the lighter ringwoodite phase producing a buoyancy force and potentially layering. Part (B) on the right hand side illustrates that the same mechanism also can work in reverse, potentially leading to layering with hot upwellings.

based on estimates of the deflection of the boundary and independent estimates of the thermal perturbations would suggest a Clapeyron slope around -2.0 to $-3.5 \mathrm{MPa} \mathrm{K}^{-1}$ (Lebedev et al., 2002; Fukao et al., 2009). We note that Liu (1994) has pointed out that the slope of the Clapeyron curve for this reaction might not be negative at higher temperatures; in that case in these regions the convection would not be layered. Hirose (2002) demonstrated experimentally that this transition might occur at around $2070 \mathrm{~K}$. This temperaturedependence to the phase transition leads to the possibility that upwellings and downwellings might be affected differently. We note that present-day estimates of mantle temperature at $660 \mathrm{~km}$ depth might be around $1880 \mathrm{~K}$ (Katsura et al., 2004); therefore, the negative Clapeyron slope could have played a dynamic role, at least for average and cold regions of the mantle, from early in Earth history.

There is also an uncertainty regarding the density contrast across this phase change, probably lying between $7.0 \%$ and 9.3\% (Dziewonski and Anderson, 1981; Weidner and Wang, 1998; Billen, 2008). The mean absolute density, in contrast, is fairly well constrained from global radial seismological models at just over $4.15 \mathrm{Kg} \mathrm{m}^{-3}$ (Dziewonski and Anderson, 1981). The coefficient of thermal expansion at around $660 \mathrm{~km}$ depth is probably slightly less than $2 \times 10^{-5} \mathrm{~K}^{-1}$ for base of upper mantle and slightly more than $2 \times 10^{-5} \mathrm{~K}^{-1}$ for uppermost lower mantle (Steinberger and Calderwood, 2006).
Considering the factors outlined above, we can reasonably expect this layering process to affect Earth's mantle dynamics. Early simulations suggest that under some conditions this layering breaks down in an episodic manner such that the evolution can be very time-dependent (Davies, 1995). This layering effect has been shown to be sensitive to the vigour of convection, with a system more likely to layer at higher vigour (Christensen and Yuen, 1985). The motivation of this work is to understand the role that such a phase change might have had on Earth's thermal and geological evolution by investigating how the vigour of convection controls the behaviour in spherical models of mantle convection.

Most work to date has focussed on 2-D, 2-D axisymmetric, and 3-D Cartesian geometries (Christensen and Yuen, 1985; Machetel and Weber, 1991; Liu et al., 1991; Peltier and Solheim, 1992; Zhao et al., 1992; Weinstein, 1993; Steinbach et al., 1993; Solheim and Peltier, 1994; Machetel et al., 1995; Peltier, 1996; Marquart et al., 2001). While there has been notable work including this phase change in spherical geometry (Tackley et al., 1993, 1994; Machetel et al., 1995; Bunge et al., 1997), there has only been limited work in spherical geometry to characterise the influence of the value of the slope of the phase change and the vigour of convection on the behaviour. Elements of our work in spherical geometry have been presented previously (Wolstencroft and Davies, 2008a,b). Yanagisawa et al. (2010) undertook a similar study simultaneously where they recently concluded that 3 domains of behaviour can be identified. Our methodology is similar: we characterise the behaviour of a large number of simulations at varying vigour of convection and strength of phase transition to define a regime diagram. We constrain the boundaries between the various behaviours and parameterise them. This allows extrapolation of the boundaries to very high vigour, which will be relevant to early Earth history but which are currently beyond computational means to simulate. After characterising the behaviour rigorously, we conclude by speculating on its possible consequences for Earth evolution.

\section{Methods}

Appropriate assumptions were made to focus on the objective of characterising how the mantle behaves as a function of both the vigour of convection and the magnitude of the negative Clapeyron slope $(\gamma)$ of the phase change reaction (i.e. the slope $\mathrm{dP} / \mathrm{dT}$ of the phase change boundary in Pressure-Temperature space). For example, in our numerical simulations we assumed the mantle is a viscous, incompressible, isochemical fluid, with constant material properties with infinite Prandtl number subject to the Boussinesq approximation (Ricard, 2007). The resulting equations modelled are: the equation for conservation of mass

$\nabla \cdot \boldsymbol{u}=0$ 
the equation for the conservation of linear momentum

$\eta \nabla^{2} \boldsymbol{u}-\nabla p+\Delta \rho g \hat{\boldsymbol{r}}=0$

and the equation for the conservation of energy

$\rho C_{p}\left(\frac{\partial T}{\partial t}+\boldsymbol{u} \cdot \nabla T\right)=k \nabla^{2} T+\rho H$

where $\boldsymbol{u}$ : velocity, $\eta$ : dynamic viscosity, $p$ : hydrodynamic pressure, $\rho$ : density, $\Delta \rho$ : lateral variation in density away from the reference state, $g$ : acceleration due to gravity, $\hat{r}$ : unit vector in radial direction, $C_{p}$ : specific heat at constant pressure, $T$ : temperature, $k$ : thermal conductivity, and $H$ : radiogenic heat production per unit mass.

Non-dimensionalisation of these equations shows that the behaviour is controlled by only 3 non-dimensional parameters: the basal heated Rayleigh number $R a$, the internal heating Rayleigh number $R a_{H}$, and the Phase Buoyancy parameter $P$ (e.g. Bunge et al., 1997).

The basal-heated Rayleigh number $R a$, is given by

$R a=\frac{\alpha \rho g \Delta T D^{3}}{\kappa \eta}$

where $\alpha$ : coefficient of thermal expansion, $\Delta T$ : superadiabatic temperature drop across the shell, $D$ : mantle thickness, and $\kappa$ : thermal diffusivity $=\frac{k}{\rho C_{p}}$.

The internal-heated Rayleigh number $R a_{H}$ is given by

$R a_{H}=\frac{\alpha \rho^{2} g H D^{5}}{\kappa k \eta}$.

Since only $\eta$ and $\gamma$ were varied, the ratio of $R a / R a_{H}=$ $\Delta T k / \rho H D^{2}=0.054$ was always constant in this work. We therefore only need one of the two Rayleigh numbers to describe our experiments; we use $R a$. This use of a fixed $R a / R a_{H}$ ratio is different to the approach adopted by Yanagisawa et al. (2010), where the $R a_{H}$ was reduced as $R a$ increased. The impact of the different treatment of $R a_{H}$ is that, in this study, the proportional contribution of the basal heat flux to total heat flux increases with $R a$. In the work of Yanagisawa et al. (2010), this proportion remains approximately constant with $R a$.

The Phase Buoyancy Parameter $P$ is given by

$P=\frac{\gamma \delta \rho}{\alpha \rho^{2} g D}$

where $\gamma$ : Clapeyron slope of the phase change, $\delta \rho$ : density change across the phase change, and $\rho$ : mean density of the two phases.

Using these equations we investigated the form of layered mantle convection for varying $R a$ and $P$. The simulations were undertaken using a benchmarked version of TERRA (Baumgardner, 1985; Bunge et al., 1997; Davies and Davies, 2009), which is able to model very high $R a$ in spherical geometry (Wolstencroft et al., 2009). The details of how TERRA solves the dimensional form of the equations with
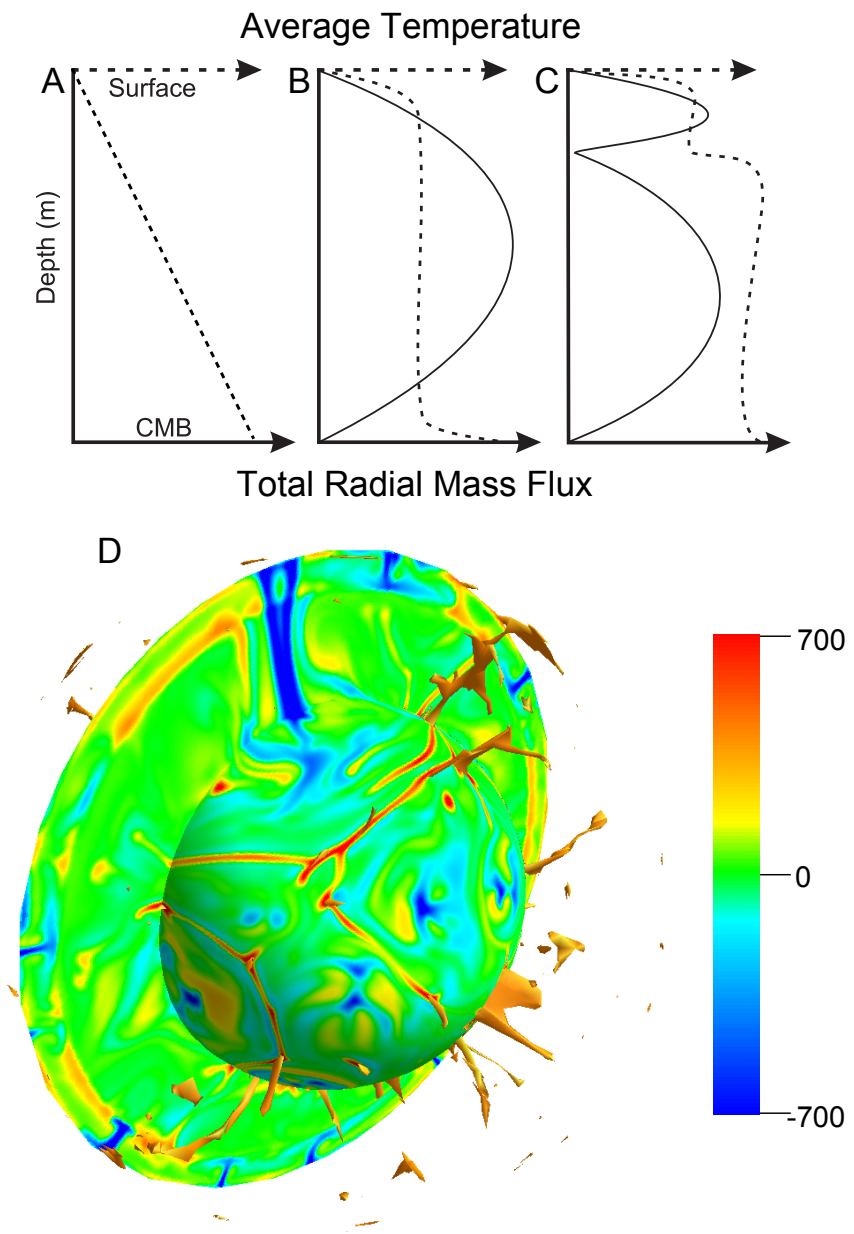

Fig. 2. Criteria for defining whether convection is layered. (A) shows the constant thermal gradient of a purely conductive regime. The dashed line in Part (B) shows the whole mantle convection thermal structure, illustrating the large temperature gradients at its boundaries. The solid line represents the absolute radial mass flux, which is zero at the two boundaries. For whole mantle convecting it peaks in mid-mantle. (C) illustrates a layered system. In this case, the solid line shows that the absolute radial mass flux will be a minimum at the phase change, which also leads to an additional thermal boundary layer, illustrated by a step in the dashed line, across the phase change. (D) shows a cross-section and a radial surface through the thermal anomaly field. Blue is colder than the radial average and red hotter. The visualisation demonstrates a transitional case. There is a large degree of layering, with the $660 \mathrm{~km}$ phase change clearly visible by temperature contrasts across the interface, but there is also some passage of material across.

pressure, temperature and velocity as the free variables, are presented in Baumgardner (1985); Bunge and Baumgardner (1995), and Yang and Baumgardner (2000). TERRA uses dimensional variables and the basic parameters of the simulations are listed in Table 1. Convection was isochemical, with free-slip and isothermal boundary conditions and a component of internal heating. Spatial resolution was carefully 
selected to resolve the features of each simulation. This resulted in the highest vigour run requiring a spatial resolution of around $15 \mathrm{~km}$ near the surface and around $22 \mathrm{~km}$ midmantle. The very lowest vigour runs only required a resolution of around $88 \mathrm{~km}$. Using a resolution appropriate to the simulation helps make this large parameter survey possible. A total of 76 simulations were undertaken, with $\gamma$ ranging from -2 to $-30 \mathrm{MPa} \mathrm{K}^{-1}$ (the equivalent phase buoyancy parameter $P$ for this range of $\gamma$ ranges from -0.0554 to -0.831 ) and $R a$ from $5.19 \times 10^{3}$ to $8.49 \times 10^{7}$ (Table 2). We note that for a sub-set of the simulations, a positive Clapeyron slope phase change was included at $410 \mathrm{~km}$ depth equal to $1.5 \mathrm{MPa} \mathrm{K}^{-1}$. Since the "660" Clapeyron slope has very large negative values, whether the small slope $410 \mathrm{~km}$ phase change is included or set to zero usually makes little difference (see Table 2). In fact at low $R a$, where this might make a difference, many simulations were undertaken with and without; no difference was found.

The phase change is implemented at a depth of $660 \mathrm{~km}$ depth using the sheet mass anomaly method of Tackley et al. (1993) implemented in TERRA by Bunge et al. (1997). This method applies an appropriate body force at the discontinuity depth, dependent on the local temperature and phase change parameters. Tackley et al. (1993) shows that this is an excellent method especially when the radial resolution cannot resolve the likely boundary deflections, as in our simulations. The sheet mass anomaly method effectively also assumes an infinitesimally thin phase loop. The sharp discontinuity observed seismically at short periods suggests that this phase change does have a thin loop (Shearer and Masters, 1992). Mineralogy experiments also suggest a thin loop (Ito and Takahashi, 1989; Wood, 1990). Earlier simulations with numerical methods including the width of the loop suggest that the large scale dynamics are largely insensitive to its width within reasonable parameter values (Christensen and Yuen, 1985; Tackley, 1995). In order to be consistent with the assumption of incompressiblity, effects related to latent heat have been disregarded. While latent heat is known to have an influence at low $R a$ (Schubert and Turcotte, 1971), with increasing $R a$, boundary deflection is known to dominate (Christensen, 1995). The work of Ita and King (1994) and Christensen and Yuen (1985) showing that Boussinesq, extended Boussinesq and compressible simulations give similar results reinforces that latent heat effects are of minor importance at high $R a$. Therefore, since we are interested in how the behaviours might extrapolate to high $R a$ (where influence of latent heat would be expected to be irrelevant), this simplification is sensible. We assume a density contrast across the phase change $\delta \rho$ of $\delta \rho / \rho \approx 9 \%$. The constancy of $\delta \rho$ implies a simple linear relationship between our Clapeyron slope $(\gamma)$ and the Phase Buoyancy Parameter $(P)$. We should remind the reader that the individual values of parameters, including $\delta \rho, \gamma$, are ultimately not important. Only the resulting values of the controlling non-dimensional parameters, $R a, R a_{H}$ and $P$, are important.
Each simulation was initiated by a radially uniform, laterally small scale, random structure. The results were independent of this initial condition since they were run for an extended length of time, until a thermal quasi-steady state was reached. This was achieved as follows. The surface and basal heat flux over time were plotted for each case. As the cases contain no evolution in heat sources, these curves show a "flattening" when balance is achieved between heat input and heat output. Cases were run until this balance was achieved. The transitional class of cases do not generally show flattening but tend to oscillate about a particular SHF value instead. Cases were classified as layered, whole mantle or transitional using the following process:

1. The total absolute radial mass flux across the mantle and the average temperature profile were plotted as shown in Fig. 2.

2. If the mass flux at $660 \mathrm{~km}$ depth was approx. $90 \%$ or greater of the maximum mass flux of the model, the case was classified whole mantle convection.

3. If the mass flux at $660 \mathrm{~km}$ depth was approx. $10 \%$ or lower than the maximum mass flux, the case was classified as layered.

4. If the case fell between these classifications and demonstrated periodic variability in surface heat flux, it was classified as transitional. The temperature profile was used to verify this state since a layered system develops a characteristic internal thermal boundary layer due to the lack of advective heat transport across the phase change (Fig. 2). Whole mantle convection shows no such internal thermal boundary layer.

5. Additionally, the cases were visualised in 3-D using isosurfaces, cross sections and specific depth surfaces (e.g. Fig. 2). These plots allow judgments to be made as to what proportion of up or down welling features penetrate the phase boundary and what the nature of the coupling is across the boundary, e.g. thermal or viscous. This final step was important for correctly classifying boundary cases where summary data values were inconclusive.

\section{Results}

The results show that at low $R a$ and high $P$ (i.e. low absolute value) whole mantle convection is preferred, while at high $R a$ and more negative $P$, layered convection is preferred (Table 2, Fig. 3). An intermediate domain of transitional behaviour is found between the two end-member behaviours. In previous work, this transitional region has been termed partially layered; we avoid this term as it does not accurately represent the wide range of behaviours displayed. To better 
Table 1. Common input parameters.

\begin{tabular}{ll}
\hline Parameter & Value \\
\hline Internal heating, $\mathrm{H}$ & $5 \times 10^{-12} \mathrm{~W} \mathrm{~kg}^{-1}$ \\
Reference density - Eq. (2), $\rho_{0}$ & $4500 \mathrm{~kg} \mathrm{~m}^{-3}$ \\
Density jump across $660 \mathrm{~km}$ phase change - Eq. $(6), \delta \rho / \rho$ & $9.1 \%$ \\
Density jump across $410 \mathrm{~km}$ phase change $\delta \rho / \rho$ & $6.4 \%$ \\
Gravitational acceleration, g & $10 \mathrm{~ms}^{-2}$ \\
Volume coefficient of thermal expansion, $\alpha$ & $2.5 \times 10^{-5} \mathrm{~K}^{-1}$ \\
Thermal conductivity, k & $4 \mathrm{~W} \mathrm{~m}^{-1} \mathrm{~K}^{-1}$ \\
Specific heat at constant volume, $C_{p}$ & $1000 \mathrm{~J} \mathrm{~K}^{-1} \mathrm{~kg}^{-1}$ \\
Temperature at outer shell boundary & $300 \mathrm{~K}$ \\
Temperature at inner shell boundary & $2850 \mathrm{~K}$ \\
Boundary conditions (velocity) & Free slip \\
Inner radius of spherical shell & $3.480 \times 10^{6} \mathrm{~m}$ \\
Outer radius of spherical shell & $6.370 \times 10^{6} \mathrm{~m}$ \\
Viscosity structure & Isoviscous \\
Pressure equation of state & Incompressible \\
Thermal equation of state & $\Delta \rho=\alpha \rho \Delta T$ \\
\hline
\end{tabular}

constrain the boundary, a large number of simulations were run at two values of $P(-0.221$ and -0.332$)$, spanning small ranges of $R a$. We have fit curves of the form $P=\alpha R a^{\beta}$ to these boundaries. The best fit curves defining our boundaries are

$P=-1.05 R a^{-0.1}$

for the boundary between the layered regime and the transitional regime, and

$P=-4.8 R a^{-0.25}$

for the boundary between the transitional regime and the whole mantle convection regime. We note that the line fits to the data are not perfect. There is a suggestion that the form of the curves, i.e. simple power law, might not be the true form of the relationship. Without guidance of an alternative relationship, we argue it is best to keep with a simple relationship, which in this case has a long history of usage in the field (Christensen and Yuen, 1985).

We now go on to compare these results with earlier work and discuss their possible implications.

\section{Discussion}

\subsection{Domains of convective mode}

As described in the Introduction, there has been a long history of investigating the effect of $R a$ and $\gamma$ on the behaviour of mantle convection with mineral phase changes. As mentioned above, our work shows three domains of behaviour: a layered convection domain at high $R a$ and more negative $P$, a whole mantle convection domain at low $R a$ and less negative $P$, and a transitional domain at intermediate values of $R a$ and $P$.

To repeat, this work focuses just on the buoyancy effect resulting from the deflection of the phase change boundary. By undertaking incompressible convection simulations, which to be self-consistent ignore the effects of the latent heat of the reaction, we are removing its influence.

We do observe episodic behaviour in the transitional domain. It is beyond the scope of this project to undertake sufficient simulations to characterise accurately where this behaviour occurs on the regime diagram, but we do note that it does not extend across the whole transitional domain and seems to occur for parameter values closer to the layered domain. It would be interesting to constrain the boundary of this behaviour.

The range of $R a$ and particularly $P$ used in this study are well beyond the likely values for Earth. Our reasons for doing this were to be able to better constrain the boundaries of the various convective domains. However, these values may have application when considering other materials (such as water ice) in other bodies such as ice planets.

\subsection{Fits to domain boundaries}

The earliest reference, to our knowledge, for a curve fit to the boundary between layered and whole mantle convection was from Christensen and Yuen (1985) 2-D Cartesian work, where the form $P=\alpha R a^{\beta}$ was assumed and the values found to be: $\alpha=-4.4$ and $\beta=-0.2$. The work presented here is similar to that of Yanagisawa et al. (2010). They also used the code TERRA and very similar input parameters to ours. The key differences between the two sets of simulations were 
Table 2. Cases modelled with case-specific parameters and outcome. Cl410: Clapeyron slope at $410 \mathrm{~km}$; Cl660: Clapeyron slope at 660 km; $P$ 660: buoyancy parameter for the 660 phase change; $R a$ : basally heated Rayleigh number; layering status classification: $1=$ whole mantle convection, 2 = two layer convection, $\mathrm{T}=$ transitional behaviour.

\begin{tabular}{|c|c|c|c|c|c|}
\hline Case & $\mathrm{Cl} 410\left(\mathrm{MPa} \mathrm{K}^{-1}\right)$ & $\mathrm{Cl} 660\left(\mathrm{MPa} \mathrm{K}^{-1}\right)$ & $P 660$ & $R a$ & Classification \\
\hline 001 & 1.5 & -2.0 & -0.0554 & $7.76 \times 10^{6}$ & 1 \\
\hline 002 & 1.5 & -4.0 & -0.111 & $7.76 \times 10^{6}$ & $\mathrm{~T}$ \\
\hline 003 & 1.5 & -4.0 & -0.111 & $1.56 \times 10^{6}$ & 1 \\
\hline 004 & 1.5 & -4.0 & -0.111 & $5.19 \times 10^{5}$ & 1 \\
\hline 005 & 1.5 & -4.0 & -0.111 & $9.74 \times 10^{4}$ & 1 \\
\hline 006 & 1.5 & -4.0 & -0.111 & $5.19 \times 10^{4}$ & 1 \\
\hline 007 & 1.5 & -4.0 & -0.111 & $1.11 \times 10^{4}$ & 1 \\
\hline 008 & 1.5 & -4.0 & -0.111 & $5.19 \times 10^{3}$ & 1 \\
\hline 009 & 1.5 & -8.0 & -0.221 & $1.56 \times 10^{6}$ & $\mathrm{~T}$ \\
\hline 010 & 1.5 & -8.0 & -0.221 & $5.19 \times 10^{5}$ & $\mathrm{~T}$ \\
\hline 011 & 1.5 & -8.0 & -0.221 & $9.74 \times 10^{4}$ & 1 \\
\hline 012 & 1.5 & -8.0 & -0.221 & $5.19 \times 10^{4}$ & 1 \\
\hline 013 & 1.5 & -8.0 & -0.221 & $1.11 \times 10^{4}$ & 1 \\
\hline 014 & 1.5 & -8.0 & -0.221 & $5.19 \times 10^{3}$ & 1 \\
\hline 015 & 1.5 & -12.0 & -0.332 & $9.74 \times 10^{4}$ & 2 \\
\hline 016 & 1.5 & -12.0 & -0.332 & $5.19 \times 10^{4}$ & $\mathrm{~T}$ \\
\hline 017 & 1.5 & -12.0 & -0.332 & $1.11 \times 10^{4}$ & 1 \\
\hline 018 & 1.5 & -12.0 & -0.332 & $5.19 \times 10^{3}$ & 1 \\
\hline 019 & 1.5 & -14.0 & -0.388 & $1.56 \times 10^{6}$ & 2 \\
\hline 020 & 1.5 & -14.0 & -0.388 & $5.19 \times 10^{5}$ & 2 \\
\hline 021 & 1.5 & -14.0 & -0.388 & $9.74 \times 10^{4}$ & 2 \\
\hline 022 & 1.5 & -14.0 & -0.388 & $5.19 \times 10^{4}$ & 2 \\
\hline 023 & 1.5 & -14.0 & -0.388 & $1.11 \times 10^{4}$ & 1 \\
\hline 024 & 1.5 & -14.0 & -0.388 & $5.19 \times 10^{3}$ & 1 \\
\hline 025 & 1.5 & -15.0 & -0.415 & $9.74 \times 10^{4}$ & 2 \\
\hline 026 & 1.5 & -15.0 & -0.415 & $5.19 \times 10^{4}$ & 2 \\
\hline 027 & 1.5 & -15.0 & -0.415 & $1.11 \times 10^{4}$ & 1 \\
\hline 028 & 1.5 & -15.0 & -0.415 & $5.19 \times 10^{3}$ & 1 \\
\hline 029 & 1.5 & -16.0 & -0.443 & $9.74 \times 10^{4}$ & 2 \\
\hline 030 & 1.5 & -16.0 & -0.443 & $5.19 \times 10^{4}$ & 2 \\
\hline 031 & 1.5 & -16.0 & -0.443 & $1.11 \times 10^{4}$ & 1 \\
\hline 032 & 1.5 & -16.0 & -0.443 & $5.19 \times 10^{3}$ & 1 \\
\hline 033 & 1.5 & -20.0 & -0.554 & $9.74 \times 10^{4}$ & 2 \\
\hline 034 & 1.5 & -20.0 & -0.554 & $5.19 \times 10^{4}$ & 2 \\
\hline 035 & 1.5 & -20.0 & -0.554 & $1.11 \times 10^{4}$ & 1 \\
\hline 036 & 1.5 & -20.0 & -0.554 & $5.19 \times 10^{3}$ & 1 \\
\hline 037 & 0.0 & -4.0 & -0.111 & $8.49 \times 10^{7}$ & $\mathrm{~T}$ \\
\hline 038 & 0.0 & -4.0 & -0.111 & $4.00 \times 10^{6}$ & 1 \\
\hline 039 & 0.0 & -4.0 & -0.111 & $2.00 \times 10^{6}$ & 1 \\
\hline 040 & 0.0 & -5.0 & -0.138 & $7.79 \times 10^{6}$ & $\mathrm{~T}$ \\
\hline 041 & 0.0 & -5.5 & -0.152 & $8.66 \times 10^{6}$ & $\mathrm{~T}$ \\
\hline 042 & 0.0 & -6.0 & -0.166 & $4.00 \times 10^{6}$ & $\mathrm{~T}$ \\
\hline 043 & 0.0 & -6.0 & -0.166 & $1.56 \times 10^{6}$ & $\mathrm{~T}$ \\
\hline 044 & 0.0 & -6.0 & -0.166 & $5.00 \times 10^{6}$ & $\mathrm{~T}$ \\
\hline 045 & 0.0 & -7.0 & -0.194 & $7.79 \times 10^{6}$ & $\mathrm{~T}$ \\
\hline 046 & 0.0 & -8.0 & -0.221 & $8.66 \times 10^{6}$ & 2 \\
\hline 047 & 0.0 & -8.0 & -0.221 & $8.00 \times 10^{6}$ & 2 \\
\hline 048 & 0.0 & -8.0 & -0.221 & $7.79 \times 10^{6}$ & 2 \\
\hline 049 & 0.0 & -8.0 & -0.221 & $6.77 \times 10^{6}$ & $\mathrm{~T}$ \\
\hline 050 & 0.0 & -8.0 & -0.221 & $5.99 \times 10^{6}$ & $\mathrm{~T}$ \\
\hline
\end{tabular}


Table 2. Continued.

\begin{tabular}{|c|c|c|c|c|c|}
\hline Case & $\mathrm{Cl} 410\left(\mathrm{MPa} \mathrm{K}^{-1}\right)$ & $\mathrm{Cl} 660\left(\mathrm{MPa} \mathrm{K}^{-1}\right)$ & $P 660$ & $R a$ & Classification \\
\hline 051 & 0.0 & -8.0 & -0.221 & $5.00 \times 10^{6}$ & $\mathrm{~T}$ \\
\hline 052 & 0.0 & -8.0 & -0.221 & $4.00 \times 10^{6}$ & $\mathrm{~T}$ \\
\hline 053 & 0.0 & -8.0 & -0.221 & $1.56 \times 10^{6}$ & $\mathrm{~T}$ \\
\hline 054 & 0.0 & -8.0 & -0.221 & $7.00 \times 10^{5}$ & $\mathrm{~T}$ \\
\hline 055 & 0.0 & -8.0 & -0.221 & $5.00 \times 10^{5}$ & $\mathrm{~T}$ \\
\hline 056 & 0.0 & -8.0 & -0.221 & $2.00 \times 10^{5}$ & $\mathrm{~T}$ \\
\hline 057 & 0.0 & -10.0 & -0.277 & $5.00 \times 10^{5}$ & $\mathrm{~T}$ \\
\hline 058 & 0.0 & -10.0 & -0.277 & $2.00 \times 10^{5}$ & $\mathrm{~T}$ \\
\hline 059 & 0.0 & -12.0 & -0.332 & $8.00 \times 10^{4}$ & 2 \\
\hline 060 & 0.0 & -12.0 & -0.332 & $7.00 \times 10^{4}$ & $\mathrm{~T}$ \\
\hline 061 & 0.0 & -12.0 & -0.332 & $6.00 \times 10^{4}$ & $\mathrm{~T}$ \\
\hline 062 & 0.0 & -12.0 & -0.332 & $5.00 \times 10^{4}$ & $\mathrm{~T}$ \\
\hline 063 & 0.0 & -12.0 & -0.332 & $4.00 \times 10^{4}$ & $\mathrm{~T}$ \\
\hline 064 & 0.0 & -12.0 & -0.332 & $2.50 \times 10^{4}$ & 1 \\
\hline 065 & 0.0 & -13.0 & -0.360 & $5.19 \times 10^{4}$ & $\mathrm{~T}$ \\
\hline 066 & 0.0 & -14.0 & -0.388 & $1.11 \times 10^{4}$ & 1 \\
\hline 067 & 0.0 & -14.0 & -0.388 & $5.19 \times 10^{3}$ & 1 \\
\hline 068 & 0.0 & -15.0 & -0.415 & $5.19 \times 10^{4}$ & 2 \\
\hline 069 & 0.0 & -15.0 & -0.415 & $2.50 \times 10^{4}$ & 1 \\
\hline 070 & 0.0 & -15.0 & -0.415 & $1.11 \times 10^{4}$ & 1 \\
\hline 071 & 0.0 & -15.0 & -0.415 & $5.19 \times 10^{3}$ & 1 \\
\hline 072 & 0.0 & -16.0 & -0.443 & $1.11 \times 10^{4}$ & 1 \\
\hline 073 & 0.0 & -16.0 & -0.443 & $5.19 \times 10^{3}$ & 1 \\
\hline 074 & 0.0 & -20.0 & -0.554 & $1.11 \times 10^{4}$ & 1 \\
\hline 075 & 0.0 & -20.0 & -0.554 & $5.19 \times 10^{3}$ & 1 \\
\hline 076 & 0.0 & -30.0 & -0.830 & $1.11 \times 10^{4}$ & 1 \\
\hline
\end{tabular}

that this study used a fixed $R a / R a_{H}$ and a $150 \mathrm{~K}$ greater temperature change across the mantle. In some cases there was also a difference in the Clapeyron slope assumed for the phase change at $410 \mathrm{~km}$ depth: 0 and $1.5 \mathrm{MPa} \mathrm{K}^{-1}$ in our work against 0 and $2.5 \mathrm{MPa} \mathrm{K}^{-1}$ in the work of Yanagisawa et al. (2010). Yanagisawa et al. (2010), like us, also define a transitional regime and their fits to the two boundaries have $\alpha=-4.7$ and $\beta=-0.2$ for the boundary between layered and transitional, and $\alpha=-12$ and $\beta=-0.33$ for the boundary between whole mantle convection and transitional. Figure 4 shows these previous domain boundaries with our curves. We note that these curves agree well at medium-high $R a$ where most of the previous data points lie, but that there are differences at lower $R a$ and in the width of the transitional domain.

Therefore, while we would not expect our and Yanagisawa et al. (2010) results to be identical (interpretation of the boundary cases might also differ), they should be sufficiently similar that it is worthwhile to consider the curves together (Fig. 4). We note that our curves also satisfy most of their points. This gives further confidence in our curves, especially as we extrapolate to high $R a$, where the Yanagisawa et al. (2010) study has many data-points.
We note that the two curves in our study converge at low Rayleigh number - we estimate this point to be around $R a \approx 2.5 \times 10^{4}$ and $P \approx-0.38$. The curves of Yanagisawa et al. (2010) do not converge at low $R a$; in fact, they have only a limited number of simulations in this range. If it is correct that the two curves should converge at low $R a$, then it tightens considerably the fit to the points and therefore the extrapolation of this relationship to high $R a$. At very high $R a$, the two curves will approach the $R a$ axis but never cross it. This is as one would expect since a mineral reaction with a non-negative Clapeyron slope cannot layer the flow with the resulting boundary deflection.

\subsection{Implications for Earth history}

\subsubsection{Limitations of modelling}

It is interesting to speculate what this research implies for mantle convective behaviour earlier in Earth history. Before doing so, we would like to emphasise that the simulations were intentionally simple to allow understanding and complete investigation of the parameter space. As a result, there are limitations when applying these outcomes to Earth. The 


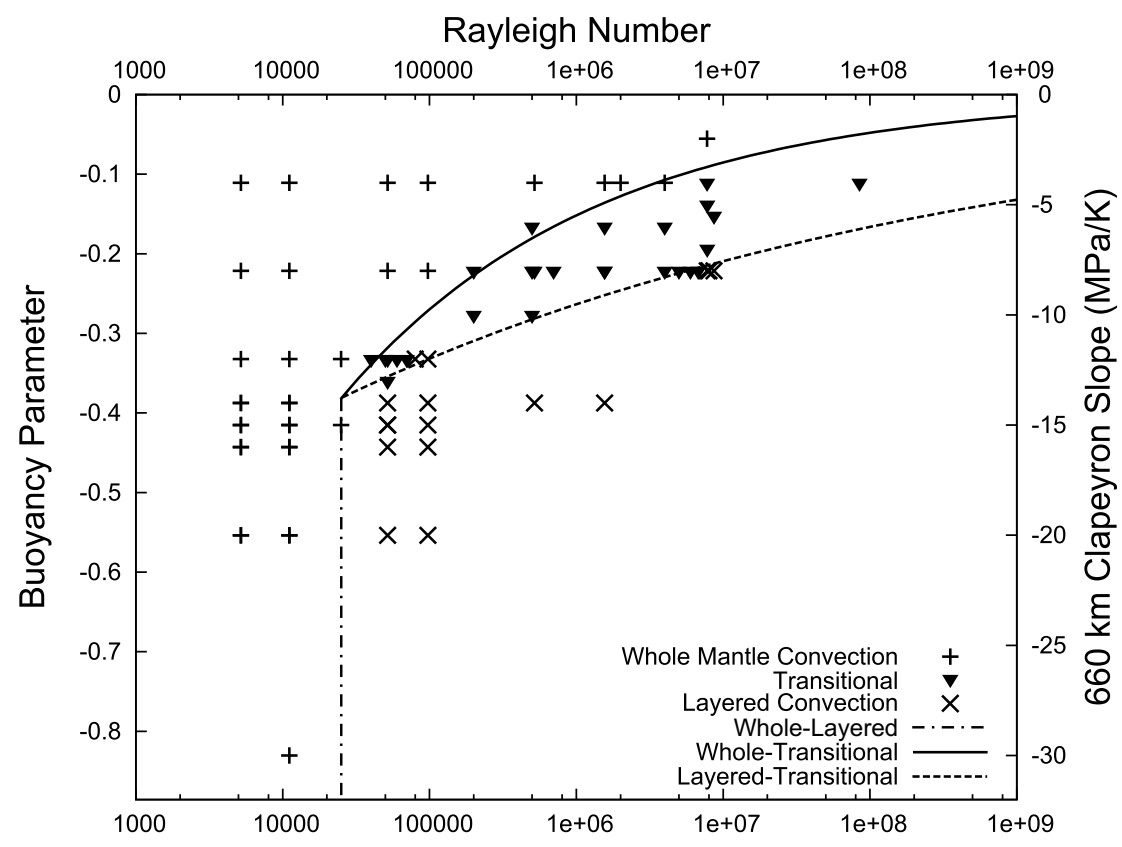

Fig. 3. Layering status of modelled cases as a function of convective vigour $(R a)$ and layering strength of the phase change (Buoyancy Parameter, $P$ ). Key defines the symbols used to indicate the nature of the convection. The solid line is a power-law fit attempting to divide the whole and transitional cases, while the dashed line divides the layered and transitional cases. The vertical dash-dot line represents the whole-layered boundary, which only occurs at very low Ra and very negative Clapeyron slope values. The equivalent Clapeyron slope for our parameters is shown on the right hand side vertical axis.

models are without depth or temperature-dependent viscosity, do not have plates or continents, and thus can not display behaviours such as slab-rollback (Goes et al., 2008; Yanagisawa et al., 2010). These simplifications could be expected to affect the detail of the conclusions here; hopefully, the broad trends in this space will remain valid as more sophisticated models are investigated in the future. More sophisticated discussions should also consider other mineral reactions, both in the olivine and garnet systems, the effect of latent heat (Christensen, 1998) and volume change (Krien and Fleitout, 2010) of the phase changes. We note that the olivine component of the mantle probably makes up around $60 \%$ of the mantle; therefore, when applying this work to the mantle the effective value of $P$ should be reduced by a similar proportion. Since there is some uncertainty regarding the proportion of olivine in the transition zone (see for example Anderson and Bass (1986) who argue for a piclogitic transition zone), our approach of not making this correction in the simulations makes it simpler for others to use this work.

\subsubsection{Rayleigh number}

Applying a single number like a Rayleigh number is clearly fraught for the actual Earth, since many properties are spatially variable and not constant. Accepting this, to advance the discussion we estimate that present day mantle $R a$ might be $\approx 10^{7}$, assuming, e.g. mean viscosity $\eta \approx 5 \times 10^{21} \mathrm{~Pa} \mathrm{~s}$, $\kappa \approx 10^{-6} \mathrm{~m}^{2} \mathrm{~s}^{-1}, \alpha \approx 2 \times 10^{-5} \mathrm{~K}^{-1}$, super-adiabatic temperature drop of $\approx 2550 \mathrm{~K}$ (Steinberger and Calderwood, 2006). Earlier in Earth history, a hotter mantle is to be expected due to the dissipation of gravitational energy from the formation era and higher radioactivity. Limited observations support this assumption that the mantle was hotter earlier in Earth history (Nisbet et al., 1995; Green, 1975). A hotter mantle would translate to a higher Rayleigh number. To illustrate the potential implications, we will assume that the only changing parameter in the $R a$ as a function of time is viscosity, with it being lower at higher temperatures. Mantle rheology is a field with large uncertainties but an activation energy of $500 \mathrm{KJ} \mathrm{mol}^{-1}$ (Korenaga and Karato, 2008) would suggest that mantle viscosity might decrease by approximately an order of magnitude for every 100 degrees increase in temperature.

Magmatic products also suggest that the mantle was hotter in the Archean, with estimates varying from $100 \mathrm{~K}$ to 300 $500 \mathrm{~K}$ hotter at $3 \mathrm{Ga}$ depending upon interpretations such as how wet the komatiite source region was and what is representative of average mantle (Lee et al., 2009; Grove and Parman, 2004; Nisbet et al., 1995; Abbott et al., 1994). Assuming a $200 \mathrm{~K}$ hotter mantle at $\approx 3 \mathrm{Ga}$, we might expect Earth to have a viscosity 2 orders of magnitude lower and therefore $R a 2$ orders of magnitude greater at $R a \approx 10^{9}$, but clearly with significant uncertainty. 


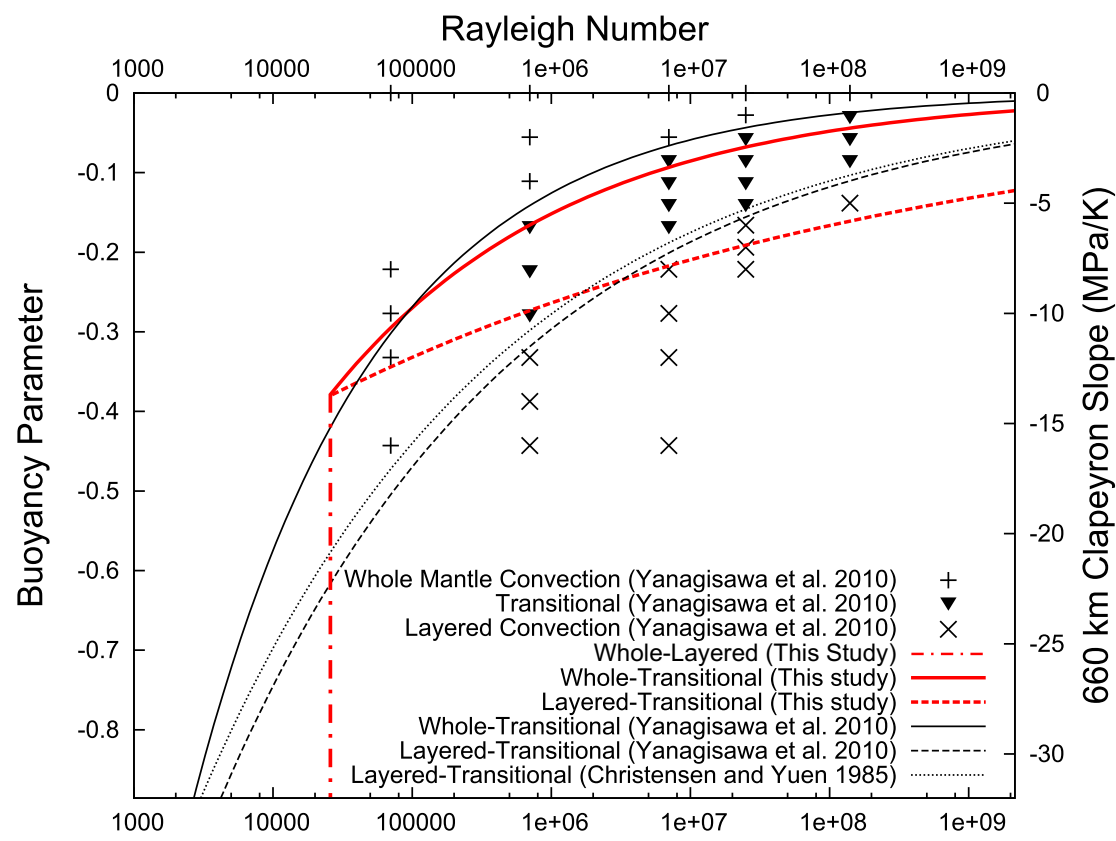

Fig. 4. Data points and curves from the study of Yanagisawa et al. (2010) with the curve from Christensen and Yuen (1985) overlaid on the domain boundary lines of this study. While we note a reasonable agreement between the points and the lines from this study, all the points do not satisfy our lines. This could be the result of the slightly different parameters and the measure of subjectivity in defining the behaviour category of a simulation. Key differences between our curves and those from previous work are the wider transitional region at high Ra and the meeting point of our curves.

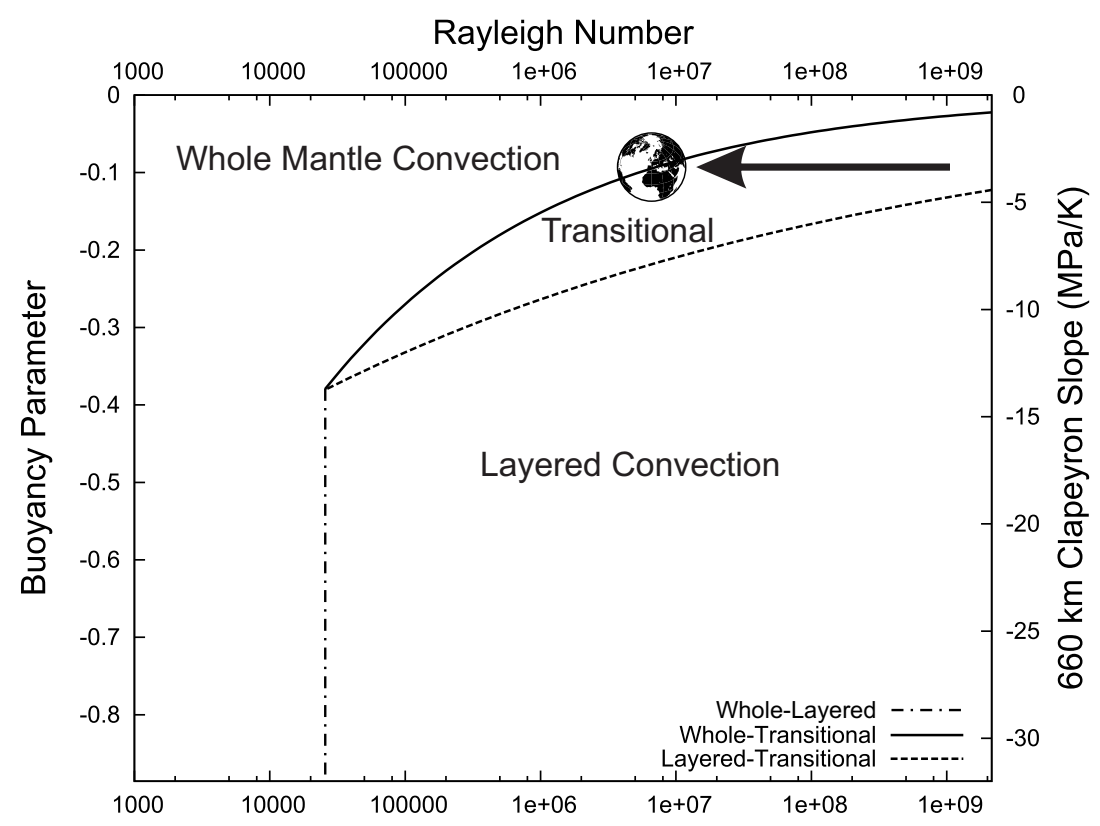

Fig. 5. The figure of Earth represents an estimate of where the present-day Earth might fit on a Rayleigh number versus Buoyancy Parameter plot. The lines mark the boundaries between the 3 regimes found in this work. The arrow shows an approximate potential mantle evolution route through the parameter space over Earth history. 


\subsubsection{Present-day Earth}

Using the estimates above for the Rayleigh number and the Phase Buoyancy parameter, we mark Earth's current position on the domain diagram (Fig. 5) with a large Earth whose size suggests an approximate sense of the uncertainty of the parameters. The approximate $R a$ and $P$ values would suggest that we are today either in the transitional regime or just in the whole mantle convection regime and very unlikely to be in the layered regime. Seismological evidence seems to strongly support this, with observations of subducting slabs descending from the upper to the lower mantle (Grand et al., 1997; van der Hilst et al., 1997; Creager and Jordan, 1984) and also observations of stagnant slabs, which might reflect some resistance at this boundary (Fukao et al., 1992)

\subsubsection{Earth evolution}

If we speculate as to where Earth was on this regime diagram $3 \mathrm{Ga}$ ago, we might expect it to be at a $R a$ approximately 2 orders of magnitude greater and similar $P$. This would suggest, in a simple interpretation, that Earth would have most likely occupied the deeper regions of the transitional domain in the past. It is possible that in very early Earth history, the mantle operated in a layered convection mode. Therefore, as we look at our results, we would predict that over Earth history the mantle evolved from a layered/transitional regime to a dominantly whole mantle convection regime. In such a scenario it is possibile that Earth might have had episodic mantle convection in its earlier history as previously suggested (Condie, 2001, 1998; Parman, 2007; Pearson et al., 2007; Frimmel, 2008; Ernst and Buchan, 2002). We also note that the evidence presented for episodicity is controversial; zircon peaks might reflect preservation (Hawkesworth et al., 2009), while it has been argued that the isotopic peaks may not be statistically robust (Rudge, 2008). Phase-change induced mantle avalanches could initiate superplumes/superevents (Condie, 1998). Such events have been suggested to affect not just magmatic outputs, but also core-generated magnetic fields (Larson, 1991). Clearly there is the potential for multiple observations to be affected. Future work to better constrain the input parameters, Earth history and undertake more realistic simulations are encouraged by the work to date.

\section{Conclusions}

We discover 3 domains of behaviour for a spherical geometry convecting mantle with a negative Clapeyron slope phase change simulating the ringwoodite to ferro-periclase and $\mathrm{Mg}$-Perovskite transition at $660 \mathrm{~km}$ depth: a whole mantle convection domain, a layered convection domain, and a transitional domain. The boundaries separating the domains converge at the low near-critical Rayleigh number, while the transitional domain (which includes episodic behaviour) is very broad at realistic Clapeyron slope. By extrapolating power law fits of these well constrained domain boundaries to high Rayleigh number (convective vigour), we suggest that it is likely that the transitional domain and possibly also the layered domain will be of interest during early Earth history and therefore for understanding Earth evolution. This work encourages more realistic simulations to be undertaken in the future as more computational resources become available.

Acknowledgements. MW would like to acknowledge the support of NERC (UK) for his studentship (NER/S/A/2005/13131). We would also like to acknowledge the support of HECToR (UK), ARCCA (Cardiff University) and Helix (Cardiff University) for computational resources. The authors would like to thank D. Rhodri Davies and the wider TERRA group for the development and maintenance of the code used in this study.

Edited by: J. van Hunen

\section{References}

Abbott, D., Burgess, L., and Longhi, J.: An empirical thermal history of the Earth's upper mantle, J. Geophys. Res., 99, 1383513850, 1994.

Anderson, D. L. and Bass, J. D.: Transition region of the Earth's upper mantle, Nature, 320, 321-328, doi:10.1038/320321a0, 1986.

Baumgardner, J. R.: Three dimensional treatment of convective flow in the Earth's mantle, J. Stat. Phys., 39, 501-511, 1985.

Bernal, J. D.: Hypothesis on the $20^{\circ}$ discontinuity, Observatory, 59, 268, 1936.

Billen, M. I.: Modeling the dynamics of subducting slabs, Annu. Rev. Earth Pl. Sc., 36, 325-356, 2008.

Bunge, H. P. and Baumgardner, J. R.: Mantle convection modeling on parallel virtual machines, Comput. Phys., 9, 207-215, 1995.

Bunge, H. P., Richards, M. A., and Baumgardner, J. R.: A sensitivity study of three-dimensional spherical mantle convection at $10^{8}$ Rayleigh number: Effects of depth-dependent viscosity, heating mode and an endothermic phase change, J. Geophys. Res., 102, 11991-12007, 1997.

Christensen, U. R.: Effects of phase transitions on mantle convection, Ann. Rev. Earth Pl. Sc., 23, 65-87, 1995.

Christensen, U. R.: Dynamic phase boundary topography by latent heat effects, Earth Planet. Sci. Lett., 154, 295-306, 1998.

Christensen, U. R. and Yuen, D. A.: Layered convection induced by phase transitions, J. Geophys. Res., 90, 10291-10300, 1985.

Condie, K. C.: Episodic continental growth and supercontinents: a mantle avalanche connection?, Earth Planet. Sci. Lett., 163, 97108, 1998.

Condie, K. C.: Mantle plumes and their record in Earth history, Cambridge University Press, Cambridge, New York, 2001.

Creager, K. C. and Jordan, T. H.: Slab penetration into the lower mantle, J. Geophys. Res., 89, 3031-3049, 1984.

Davies, G. F.: Punctuated tectonic evolution of the Earth, Earth Planet. Sci. Lett., 136, 363-379, 1995.

Davies, D. R. and Davies, J. H.: Thermally-driven mantle plumes reconcile multiple hot-spot observations, Earth Planet. Sci. Lett., 278, 50-54, doi:10.1016/j.eps1.2008.11.027, 2009. 
Dziewonski, A. and Anderson, D. L.: Preliminary reference Earth model, Phys. Earth Planet. Int., 25, 297-356, 1981.

Ernst, R. E. and Buchan, K. L.: Maximum size and distribution in time and space of mantle plumes: evidence from large igneous provinces, J. Geodyn., 34, 309-342, 2002.

Fei, Y., Van Orman, J., Li, J., van Westrenen, W., Sanloup, C., Minarik, W., Hirose, K., Komabayashi, T., Walter, M., and Funakoshi, K.: Experimentally determined postspinel transformation boundary in $\mathrm{Mg}_{2} \mathrm{SiO}_{4}$ using $\mathrm{MgO}$ as an internal pressure standard and its geophysical implications, J. Geophys. Res., 109, 2004.

Frimmel, H. E.: Earth's continental crustal gold endowment, Earth Planet. Sci. Lett., 267, 45-55, 2008.

Fukao, Y., Obayashi, M., Inoue, H., and Nenbai, M.: Subducting slabs stagnant in the mantle transition zone, J. Geophys. Res., 97, 4809-4822, 1992.

Fukao, Y., Obayashi, M., Nakakuki, T., and Deep Slab Project, A.: Stagnant slab: A review, Annual. Rev. Earth Pl. Sc., 37, 19-46, 2009.

Goes, S., Capitanio, F. A., and Morra, G.: Evidence of lower-mantle slab penetration phases in plate motions, Nature, 451, 981-984, 2008.

Grand, S. P., Hilst, R. D. v. d., and Widiyantoro, S.: Global Seismic Tomography: A Snapshot of Convection in the Earth, GSA Today, 7, 1-7, 1997.

Green, D. H.: Genesis of Archean peridotitic magmas and constraints on Archean geothermal gradients and tectonics, Geology, 3, 15-18, 1975.

Grove, T. and Parman, S. W.: Thermal evolution of the Earth as recorded in komatiites, Earth Planet. Sci. Lett., 219, 173-187, 2004.

Hawkesworth, C., Cawood, P., Kemp, T., Storey, C., and Dhuime, B.: A matter of preservation, Science, 323, 49-50, 2009.

Hirose, K.: Phase transitions in pyrolitic mantle around $670-\mathrm{km}$ depth: Implications for upwelling of plumes from the lower mantle, J. Geophys. Res., 107, B2078, doi:10.1029/2001JB000,597, 2002.

Irifune, T., Nishiyama, N., Kuroda, K., Inoue, T., Isshiki, M., Utsumi, W., Funakoshi, K., Urakawa, S., Uchida, T., Katsura, T., and Ohtaka, O.: The postspinel phase boundary in $\mathrm{Mg}_{2} \mathrm{SiO}_{4}$ determined by in situ X-ray diffraction, Science, 279, 1698-1700, 1998.

Ita, J. and King, S.: The sensitivity of convection with an endothermic phase change to the form of governing equations, initial conditions, boundary conditions, and equation of state, J. Geophys. Res., 99, 15919-15938, 1994.

Ito, E. and Takahashi, E.: Postspinel transformations in the system $\mathrm{Mg}_{2} \mathrm{SiO}_{4}-\mathrm{Fe}_{2} \mathrm{SiO}_{4}$ and some geophysical implications, J. Geopys. Res., 94, 10637-10646, 1989.

Katsura, T., Yamada, H., Shinmei, T., Kubo, A., Ono, S., Kanzaki, M., Yoneda, A., Walter, M. J., Ito, E., Urakawa, S., Funakoshi, K., and Utsumi, W.: Post-spinel transition in $\mathrm{Mg}_{2} \mathrm{SiO}_{4}$ determined by high P-T in situ X-ray diffractometry, Phy. Earth Planet. In., 136, 11-24, 2003.

Katsura, T., Yamada, H., Nishikawa, O., Song, M., Kubo, A., Shinmei, T., Yokoshi, S., Aizawa, Y., Yoshino, T., Walter, M. J., Ito, E., and Funakoshi, K.: Olivine-wadsleyite transition in the system $(\mathrm{Mg}, \mathrm{Fe})_{2} \mathrm{SiO}_{4}$, J. Geophys. Res., 109, B02209 doi:10.1029/2003JB002438, 2004.
Korenaga, J. and Karato, S.: A new analysis of experimental data on olivine rheology, J. Geophys. Res., 113, B02403, doi:10.1029/2007JB005100, 2008.

Krien, Y. and Fleitout, L.: Accommodation of volume changes in phase transition zones : Macroscopic scale, J. Geophys. Res., 115, B03403, doi:10.1029/2009jb006505, 2010.

Larson, R. L.: Geological consequences of superplumes, Geology, 19, 963-966, 1991.

Lebedev, S., Chevrot, S., and van der Hilst, R. D.: Seismic evidence for olivine phase changes at the 410- and 660-kilometer discontinuities, Science, 296, 1300-13-2, 2002.

Lee, C.-T. A., Luffi, P., Plank, T., Dalton, H., and Leeman, W. P.: Constraints on the depths and temperatures of basaltic magma generation on Earth and other terrestrial planets using new thermobarometers for mafic magmas, Earth Planet. Sci. Lett., 279, 20-33, 2009.

Litasov, K., Ohtani, E., Sano, A., Suzuki, A., and Funakoshi, K.: In situ X-ray diffraction study of postspinel transformation in a peridotite mantle: implication for the 660-km discontinuity, Earth Planet. Sci. Lett., 238, 311-328, 2005.

Liu, M.: Asymmetric phase effects and mantle convection patterns, Science, 264, 1904-1907, 1994.

Liu, M., Yuen, D. A., Zhao, W., and Honda, S.: Development of diapiric structures in the upper mantle due to phase transitions, Science, 252, 1836-1839, 1991.

Machetel, P. and Weber, P.: Intermittent layered convection in a model mantle with an endothermic phase-change at $670 \mathrm{~km}, \mathrm{Na}-$ ture, 350, 55-57, 1991.

Machetel, P., Thoraval, C., and Brunet, D.: Spectral and geophysical consequences of 3-D spherical mantle convection with an endothermic phase change at the $670 \mathrm{~km}$ discontinuity, Phys. Earth Planet. In., 88, 43-51, 1995.

Marquart, G., Schmeling, H., Ito, G., and Schott, B.: Conditions for plumes to penetrate the mantle phase boundaries, J. Geophys. Res., 105, 5679-5693, 2001.

Nisbet, E. G., Cheadle, M. J., Arndt, N. T., and Bickle, M. J.: Constraining the potential temperature of the Archean mantle: a review of the evidence from komatiites, Lithos, 30, 291-307, 1995.

Ohtani, E. and Litasov, K. D.: The effect of water on mantle phase transitions, in: Water in Nominally Anhydrous Minerals, 62, Rev. Mineral. Geochem., 397-419, Mineralogical Society of America, 2006.

Olson, P. and Yuen, D.: Thermochemical plumes and mantle phase transitions, J. Geophys. Res., 87, 3993-4002, 1982.

Parman, S. W.: Helium isotopic evidence for episodic mantle melting and crustal growth, Nature, 446, 900-903, 2007.

Pearson, D. G., Parman, S. W., and Nowell, G. M.: A link between large mantle melting events and continent growth seen in Osmium isotopes, Nature, 449, 202-205, 2007.

Peltier, W. R.: Phase-transition modulated mixing in the mantle of the Earth, Philos. T. Roy. Soc. A, 354, 1425-1447, 1996.

Peltier, W. R. and Solheim, L. P.: Mantle phase transitions and layered chaotic convection, Geophys. Res. Lett., 19, 321-324, 1992.

Ricard, Y.: Physics of Mantle Convection, in: Treatise of Geophysics, 7, Elsevier, 2007.

Ringwood, A. E.: Phase transformations in the mantle, Earth Planet. Sci. Lett., 5, 401-412, 1969.

Rudge, J. F.: Finding peaks in geochemical distributions: A reexamination of the helium-continental crust correlation, Earth 
Planet. Sci. Lett., 274, 179-188, 2008.

Schubert, G. and Turcotte, D. L.: Phase transitions and mantle convection, J. Geophys. Res., 76, 1424-1432, 1971.

Shearer, P. M. and Masters, G. M.: Global mapping of topography on the 660-km discontinuity, Nature, 355, 791-796, 1992.

Shim, S.-H., Duffy, T. S., and Shen, G.: The post-spinel transformation in $\mathrm{Mg}_{2} \mathrm{SiO}_{4}$ and its relation to the $660-\mathrm{km}$ discontinuity, Nature, 411, 571-574, 2001.

Solheim, L. P. and Peltier, W. R.: Avalanche effects in phasetransition modulated thermal-convection - a model of Earth's mantle, J. Geophys. Res., 99, 6997-7018, 1994.

Steinbach, V., Yuen, D. A., and Zhao, W.: Instabilities from phase transitions and the timescales of mantle thermal convection, Geophys. Res. Lett., 20, 1119-1122, 1993.

Steinberger, B. and Calderwood, A. R.: Models of large-scale viscous flow in the Earth's mantle with constraints from mineral physics and surface observations, Geophys. J. Int., 167, 1461-1481, 2006.

Tackley, P. J.: On the penetration of an endothermic phase transition by upwellings and downwellings, J. Geophys. Res., 100, 1547715488, 1995.

Tackley, P. J., Stevenson, D. J., Glatzmaier, G. A., and Schubert, G.: Effects of an endothermic phase change at $670 \mathrm{~km}$ depth in a spherical model of convection in the Earth's mantle, Nature, 361, 699-704, 1993.

Tackley, P. J., Stevenson, D. J., Glatzmaier, G. A., and Schubert, G.: Effects of multiple phase transitions in a three dimensional spherical model of convection in Earth's mantle, J. Geophys. Res., 99, 15877-15901, 1994.

van der Hilst, R., Widiyantoro, S., and Engdahl, E. R.: Evidence for Deep Mantle Circulation from Global Tomography, Nature, 386, 578-584, 1997.

Weidner, D. and Wang, Y.: Chemical- and Clapeyron-induced buoyancy at the $660 \mathrm{~km}$ discontinuity, J. Geophys. Res., 103, 74317441, 1998.
Weinstein, S. A.: Catastrophic overturn of the Earth's mantle driven by multiple phase changes and internal heat generation, Geophys. Res. Lett., 20, 101-104, 1993.

Wolstencroft, M. and Davies, J. H.: Time Dependent Layering in Earths Mantle: Mantle Avalanches and Thermal Pulses, EOS Trans, AGU, 89(53), 2008a.

Wolstencroft, M. and Davies, J. H.: The influence of convective vigour on phase change induced layering at $660 \mathrm{~km}$ in early Earth's mantle, Geophys. Res. Abstr., EGU2008-A, 00228, 2008b.

Wolstencroft, M., Davies, J. H. and Davies D. R.: Nusselt-Rayleigh number scaling for spherical shell Earth mantle simulation up to a Rayleigh number of $10^{9}$, Phys. Earth Planet. Int., 176, 132141, 2009.

Wood, B. J.: Postspinel transformations and the width of the $670 \mathrm{~km}$ discontinuity: A comment on "Postspinel transformations in the system $\mathrm{Mg}_{2} \mathrm{SiO}_{4}-\mathrm{Fe}_{2} \mathrm{SiO}_{4}$ and some geophysical implications" by E. Ito and E. Takahashi, J. Geophys. Res., 95, 12681-12685, 1990.

Yanagisawa, T., Yamagishi, Y., Hamano, Y., and Stegman, D. R.: Mechanism for generating stagnant slabs in 3-D spherical mantle convection models at Earth-like conditions, Phys. Earth Planet. Int., 183, 342-352, 2010.

Yang, W. S. and Baumgardner, J. R.: Matrix-dependent transfer multigrid method for strongly variable viscosity infinite Prandtl number thermal convection, Geophys. Astrophys. Fluid Dyn., 92, 151-195, 2000.

Zhao, W., Yuen, D. A., and Honda, S.: Multiple phase transitions and the style of mantle convection, Phys. Earth Planet. Int., 72, 185-210, 1992. 